\title{
Avaliação da adequação técnica de indústrias de medicamentos fitoterápicos e oficinais do Estado do Rio de Janeiro
}

\author{
Assessment of the technical adequacy of phytotherapeutics \\ and officinal medicine manufacturers in the State of Rio de Janeiro
}

\author{
Natália Dias da Costa Alves ${ }^{1}$ \\ Tereza Cristina dos Santos ${ }^{1}$ \\ Carlos Rangel Rodrigues ${ }^{2}$ \\ Helena Carla Castro ${ }^{3}$ \\ Luiz M arcelo Lira ${ }^{4}$ \\ Camila Braga D ornelas ${ }^{5}$ \\ Lúcio M endes Cabral ${ }^{4}$
}

\footnotetext{
${ }^{1}$ Secretaria Estadual de Vigilância Sanitária do Rio deJaneiro, Centro de Vigilância Sanitária Estadual. Rua M éxico 128/ 3o andar, sala 231 Castelo. 20231-031 Rio de Janeiro RJ.ndcalves@hotmail.com 2 M odM olQSAR, Faculdade deFarmácia, Universidade Federal do Rio de Janeiro.

${ }^{3}$ LabioM ol, Instituto Biologia, Universidade Federal Fluminense.

${ }^{4}$ LabTecFarm, Faculdadede Farmácia, Universidade Federal do Rio de Janeiro. 5 Instituto de

Abstract In this study we evaluated the current profile of manufacturers of officinal medicinesand phytotherapeutics in relation to the Brazilian regulatory legislation. Forty-eight industries involved in manufacture and distribution of officinal medicines and phytotherapeutics were identified in one hundred current administrative processes of the $\mathrm{N}$ ational $\mathrm{H}$ ealth Surveillance A gency in Brazil. The analysis of the inspection reports of these companies considered the first nine months after theimplantation of Resolution RDC 210/03 and revealed five specific company profiles: satisfactory $(29.2 \%)$, satisfactory with restrictions $(10.4 \%)$, unsatisfactory $(6.2 \%)$, interdicted $(39.6 \%)$, and applying for cancellation due to lack of conditions for complying with Resolution RDC 210/03 (14.6\%). The main irregularities found in these companies involved the operational flow, quality control and product registration issues. O ur results revealed a great number of companies whose activities are related to the production of officinal medicines and phytotherapeutics but that are still not totally adequate to the industrial park of Rio de Janeiro. The perspectives for the future of this category of industries are thus not favorable because of their difficulties to meet the current requirements.

Key words Phytotherapeutics, Brazilian National $\mathrm{H}$ ealth Surveillance Agency -ANVISA, Drug registration, Good manufacturing practices
Resumo N este trabalho, buscou-sedeterminar o perfil de indústriasfarmacêuticas dedicadas à fabricação e fracionamento de medicamentos fitoterápicos e oficinais no Estado do Rio de Janeiro por informações do CVS-RJ. Foram identificadas 48 empresas com atividades relacionadas à fabricação e/ou fracionamento e distribuição de me dicamentos oficinais e fitoterápicos e analisadas no grau de adequação à legislação sanitária vigente, cumprimento de boas práticas de fabricação vigentes, bem como às questões relacionadas ao registro de produtos; as principais irregularidades foram o fluxo depessoal econtroledequalidadeadequado. Constatou-se que há atualmente empresas em situação: satisfatória $(29,2 \%)$, satisfatórias com restrições $(10,4 \%)$, insatisfatórias $(6,2 \%)$, interditadas $(39,6 \%)$, e solicitantes do cancelamento do processo por não terem condições para o cumprimento da RDC n०210/03 $(14,6 \%)$. Segundo o quadro atual, ainda égrande o número de empresas com atividades relacionadas à fabricação e/ou fracionamento e distribuição de medicamentos fitoterápicos e oficinais em fase de adequação ou em condições não adequadas no Rio de aneiro. Ao mesmo tempo, é pouco factível a adequação, frente ao tipo de produto e porte financeiro, apontando um prognóstico desfavorável para o setor em questão.

Palavras-chave M edicamentos fitoterápicos, AN VISA, Registro de medicamentos, Boas práticas de fabricação 
Introdução

A fitoterapia representa uma alternativa histórica ao uso de medicamentos sintéticos, geralmente considerados mais caros e agressivos ao organismo, mas que, ao contrário do que se especula, pode oferecer riscos à saúde quando não utilizada de forma adequada. Um conceito, muitas vezes irreal, é o baixo custo desta terapia, visto que alguns fitoterápicos, dependendo da complexidade de sua produção, podem ter custos similares aos dos produtos sintéticos. Entretanto, a principal justificativa para o uso desta categoria de produtos se refere ao menor custo do seu desenvolvimento e ao fato de quea grande maioria não seencontra sob proteção patentária, tornando-os uma alternativa terapêutica bastante promissora para países pobres ou em desenvolvimento como o Brasil em vista de possuir um terço da flora mundial ${ }^{18}$. Ao contrário da tradição popular que as classifica como sendo naturais e isentas de reações adversas, o uso de preparações fitoterápicas pode conduzir a diversos tipos de danos à saúde, como reações alérgicas, tóxicas, efeitos mutagênicos einterações medicamentosas $3,5,7,8,9,10,11,12,13,15,17$.

A literatura mostra exemplos como o uso tradicional há mais de mil anos de Jin Bu Huan, uma planta chinesa que apresenta um efeito analgésico e sedativo, e cujo emprego está relacionado a sete casos de hepatite severa após sua utilização ${ }^{7}$. Algumas espécies de plantas medicinais têm alta toxicidade dependente da via de administração utilizada, como o confrei (Symphytum officinale L.), cujo chá e suco já foram largamente utilizados com fins terapêuticos; contudo, estudos científicos comprovaram que esta espécie sintetiza alcalóides pirrolizidínicos, substâncias causadoras de necrose centro-lobular, levando a lesões hepáticas graves. H oje, o uso do confrei se limita a produtos de uso externo de aplicação tópica ${ }^{14}$. Estes exemplos, dentreinúmeros outros, nosleva a concluir queo uso tradicional deplantas medicinais pela população não é garantia de segurança do seu uso como medicamento, embora a Organização Mundial de Saúde (OMS) tenha divulgado que $65-80 \%$ da população dos países em desenvolvimento dependiam das plantas medicinais como única forma de acesso aos cuidados básicos de saúde1.

No Brasil, o medicamento fitoterápico está definido pela Agência Nacional de Vigilância Sanitária (ANVISA) como sendo medicamentos obtidos a partir de plantas medicinais. Eles são obtidos empregando-se exclusivamente derivados de droga vegetal (extrato, tintura, óleo, cera, exsudato, suco, e outros). Não é objeto de registro como medicamento fitoterápico, planta medicinal ou suas partes, após processos de coleta, estabilização e secagem, podendo ser íntegra, rasurada, triturada ou pulverizada. Este medicamento é caracterizado pelo conhecimento da sua eficácia e dos riscos de seu uso, assim como pela reprodutibilidade garantia de sua qualidade 4 . Não se considera medicamento fitoterápico aquele que, na sua composição, inclua substâncias ativas isoladas, de qualquer origem, nem as associações destas com extratos vegetais ${ }^{4}$. Torna-se evidente, então, a necessidade de uma efetiva fiscalização deste setor, sem a qual pode se deparar com sérios problemas de saúde pública. Em seu artigo 76, o Decreto № 20.377/31 determinava a apreensão e inutilização de plantas medicinais sob classificações botânicas falsas ou desprovidas de ação terapêutica. Esta é uma determinação correta, mas que demonstra a ambigüidade entre os termos identificação, mais adequado ao sentido pretendido na legislação, do que o termo classificação botânica. Ao final, seguiam-se listas de produtos que poderiam existir nas farmácias, citando inúmeras drogas e insumos derivados, tais como: abacateiro, abútua, açafrão, ácido gálico, boldo, camomila, cânfora, mirra, ipeca, valeriana e outras².

A ausência deum sistema de fiscalização efetivo, associado ao momento de competição acirrada entre as indústrias nacionais e multinacionais quecomercial izavam medicamentosfitoterápicos, propiciou condições ao surgimento edesenvolvimento de empresas pouco estruturadas, o que levou à fraude e má qualidade dos produtos. Este quadro também caracterizou a incapacidade dos órgãos governamentais em implantar a legislação na prática. Em agosto de 2003, a ANVISA publicou a Resolução de Diretoria Colegiada (RDC) № 210, cuja redação estabelece as bases das Boas Práticas de Fabricação de M edicamentos, bem como traz em um de seus anexos um roteiro orientativo de inspeção para estabelecimentos que exerçam tal atividade, aplicando-se este instrumento de forma indiferenciada para todas as empresas produtoras de medicamentos, sendo estes fitoterápicos ou não. Dentro deste contexto, esteinstrumento normativo vem sendo utilizado para a realização de inspeções nas indústrias de medicamentos fitoterápicos e oficinais existentes no Estado do Rio de Janeiro. Com a maior ênfase da fiscalização da indústria farmacêutica direcionada pela ANVISA, os medicamentosfitoterápicos vêm ganhando relevância no mercado e no Sistema Nacional de Vigilância Sa- 
nitária, tendo em vista ainda o crescimento do consumo dos mesmos no Brasil e no mundo. N esta nova realidade, se avalia atualmente o parqueindustrial farmacêutico dedicado à produção de fitoterápicos ${ }^{18}$. A RDC n 210/03, em consonância com a Lei Federal no 6.360/76 ( quedispõe sobre a vigilância sanitária a que ficam sujeitos os medicamentos, as drogas, os insumos farmacêuticos e correlatos, cosméticos, saneantes e outros produtos), demonstra claramente a importância deum sistema eficaz de monitoramento sanitário para toda e qual quer indústria que produza e/ou fracione medicamentos.

Com base nesse cenário então descrito, tomou-se como objetivo principal deste trabalho avaliar a situação mercadológica atual das indústrias farmacêuticas produtoras de medicamentos fitoterápicos e oficinais, assim como a adequação das mesmas frente às boas práticas e fabricação vigentes, utilizando como basea resolução RDC n² 210/03, buscando detectar a presente situação para este segmento do mercado, tanto em termos sanitários quanto em termos de sua subsistência.

\section{Metodologia}

\section{Origem dos dados}

Após a estruturação organizacional da Vigilância Sanitária do Estado do Rio de Janeiro, foram iniciadas, pelo Setor de M edicamentos do Departamento deFiscalização deM edicamento e Afins do Centro de Vigilância Sanitária do Estado, inspeções sanitárias baseadas nas linhas de atividades exercidas pelas empresas. N essa ótica, a partir do estudo dos processos administrativos existentes para cada empresa no Centro Vigilância Sanitária do Estado do Rio de Janeiro, e das informações disponibilizadas pela ANVISA, foi realizado um levantamento de dados objetivando o conhecimento do quantitativo de empresas fabricantes e/ou fracionadoras de medicamentosfitoterápicos e oficinais existentes, bem como a situação das mesmas frente à legislação vigente, no que tange às exigências de adequação da planta produtiva e/ou atendimento de requisitos técnicos/tecnológicos. Para tal, foram utilizados os processos administrativos existentes para cada empresa, constituídos por toda documentação legal da empresa desde o início desuas atividades, bem como das plantas arquitetônicas e relatórios de inspeções sanitárias realizadas, o queindica a situação das mesmas. Foi ava- liado um total de cem processos sanitários, direcionando-se a análise para todas as empresas situadas no Estado do Rio de Janeiro dedicadas à produção destas classes de medicamentos.

\section{Organização dos dados}

Os dados foram organizados com auxílio de tabelas e gráficos, demonstrando o quantitativo atual de empresas fabricantes de medicamentos fitoterápicos e oficinais encontradas em nosso parque industrial, o comportamento delas frenteàs exigências técnicas, a partir da implementação das legislações regulamentatórias da área, e as principais características e pontos críticos observados segundo as variáveis adotadas.

\section{Classificação da amostra \\ e análise de dados}

As empresas fabricantes e/ou fracionadoras de medicamentos fitoterápicos e oficinais encontradas no parque industrial foram classificadas quanto às seguintes variáveis: localização por regiões do Estado do Rio de Janeiro, porte da empresa, atividades exercidas e categorias de produtosfabricados. Os dadosforam analisados a partir da correlação entre a classificação proposta para a amostra, o quantitativo de empresas existente, os pontos críticos encontrados, as adequações e 0 número de inspeções realizadas e a situação atual encontrada. Para nortear as conclusões retiradas do estudo do material analisado, foi efetuado um estudo complementar baseado nos dados obtidos a partir do levantamento das denúncias recebidas e apuradas pelo Setor de M edicamentos do Centro de Vigilância Sanitária, relacionadas a estas classes específicas de medicamentos, traçando-se um paralelo entre o grau de adequação de boas práticas de fabricação da empresa e a maior incidência de denúncias de desvios e reações adversas para as mesmas.

\section{Resultados ediscussão}

Atualmente, no Estado do Rio de Janeiro, existem 189 indústrias de medicamentos, incluindo as farmoquímicas e as indústrias importadoras e distribuidoras de medicamentos, que realizam o fracionamento de produtos. N este universo, a partir da análise dos processos administrativos de cada empresa existente no Setor de M edicamentos do Centro de Vigilância Sanitária do Estado do Rio de Janeiro (CVS/SES/RJ) e dos regis- 
tros de produtos concedidos a cada uma delas, foram identificadas 48 empresas com atividades relacionadas à fabricação e/ou fracionamento e distribuição de medicamentos fitoterápicos eoficinais no estado. Considerando a variável localização geográfica, ea partir dos endereços existentes nos processos administrativos de cada empresa fabricante e/ou fracionadora de medicamentos fitoterápicos e oficinais analisados, foi observada uma maior concentração destas empresas na região metropolitana do Rio de Janeiro, compreendendo vinte municípios do estado. Foram totalizadas 36 empresas exercendo as atividades em questão, sendo que $77 \%$ localizadas no próprio município do Rio de Janeiro. $\mathrm{N}$ as demais regiões, poucas empresas foram identificadas, sendo que após o recebimento de denúncias, duas empresas foram identificadas funcionando clandestinamente no município de Campos dos Goytacazes e em Conceição de M acabu. Esta concentração de empresas junto à metrópole do estado se justifica por uma mai or facilidade de acesso a informações técnicas e pessoal qualificado, não sendo, portanto esperado deficiências neste sentido na grande maioria das empresas analisadas, salvo as localizadas nas regiões mais distantes da capital.

A localização geográfica pode contornar alguns entraves tecnológicos; porém, torna-se ainda necessário avaliar a capacidadefinanceira destas empresas para viabilizar o exercício contínuo de suas atividades segundo a legislação sanitária vigente. Desta forma, a partir da análise dos contratos sociais e do Cadastro Nacional de Pessoa Jurídica (CNPJ) das empresas, alvos desta avaliação, foi possível verificar o porte das mesmas. Segundo os parâmetros adotados pela ANVISA, considera-se uma empresa de pequeno porte àquela cujo faturamento anual situa-se entre $R \$$ $500.000,00$ (quinhentosmil reais) a $\mathrm{R} \$ 1.000 .000,00$ (hum milhão de reais), enquanto as de médio porte seriam aquelas com faturamento anual variando de R \$1,2 milhão (hum milhão eduzentos mil reais) a $\$ 15$ milhões (quinzemilhões). Pelos dados obtidos, pode se concluir que a grande maioria das empresas do segmento estudado é representada por empresas de pequeno porte, 0 que sugere uma restrição às adequações técnicas em função dos altos custos, dificultan do a implementação das normas de boas práticas de fabricação. Visto que o custo médio de construção/ adequação de uma indústria produtora de medicamentosécerca de $R \$ 800.000,00$ (oitocentosmil reais) por metro quadrado, a adequação de seiscentos metros quadrados (média de tamanho para empresas de pequeno porte) representaria um ônus de $R \$ 480.000,00$ (quatrocentos eoitenta mil reais) além do valor de aquisição de equipamentos e validação da planta, que pode encarecer ainda mais este investimento ${ }^{6}$. Gera-se então uma contradição em que a negligência do sistema regulador sanitário no passado possibilitou a subsistência de empresas quenão possuem condições técnico-financeiras para funcionar como uma indústria farmacêutica dentro de padrões sanitários mínimos. Atualmente, o maior rigor regulatório vigente permiteaidentificação esta deficiência, levando ao fechamento ou funcionamento irregular de muitas destas empresas. Não se pode, contudo, justificar esta realidade pela característica do medicamento fitoterápico, seja nos aspectos técnico ou mercadológico, visto que 0 líder de mercado do seguimento, o Vick Vaporub $\otimes$, desenvolvido por uma empresa multinacional, fatura em média US $\$ 20$ milhões (vinte miIhões de dólares) por ano. Cabe ressaltar ainda queo mercado de fitoterápicos, formado hojepor cerca de duzentoslaboratóriosfabricantesno país, movimenta em torno de US\$ 400 milhões (quatrocentos milhões de dólares) e representa apenas $6,7 \%$ das vendas de medicamentos no Brasil, sendo um mercado promissor e em franca expansão. Outros produtos como o Tebonim ${ }^{\circledR}$,

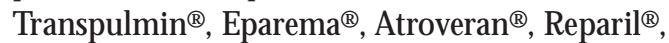

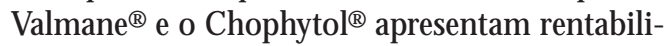
dades bastante elevadas, desmistificando de forma definitiva a ilegalidade deste mercado ou sua poucasignificânciafinanceira.

Quanto ao segmento de medicamentos oficinais, não existe uma característica de mercado clara nem uma marca vinculada aos mesmos, 0 que reduz seu retorno financeiro. Ainda se soma a este viés o desenvolvimento de produtos com marca própria por redes de farmácias ou drogarias independentes, o que acaba caracterizando este mercado como marginal para a indústria de medicamentose, portanto, de difícil subsistência frente aos parâmetros sanitários atuais.

$\mathrm{N}$ a avaliação da adequação das empresas estudadas em termos das boas práticas de fabricação, pelo uso do instrumento legal, RDC n²10/ 03, foi observado um cenário bastante desfavorável, que se agrava ainda mais quando se avalia o universo das empresas classificadas como satisfatórias, representadas por apenas 29\% (Tabela 1). Grande parte destas empresas não se dedica exclusivamente à produção de medicamentos fitoterápicos ou oficinais, havendo, inclusive, duas multinacionais neste total. Poderia se afirmar que a dificuldade de adequação das 
empresas produtoras é uma peculiaridade do produto fitoterápico. Porém, ao se comparar às resoluções publicadas pela ANVISA para registro de medicamentos fitoterápicos e alopáticos, se verifica uma similaridadetécnica nas exigências pertinentes, em quase sua totalidade, como pode ser visto na Tabela 2. Analisando-se detaIhadamente a legislação vigente, pode se observar uma maior flexibilidade no registro de produtos fitoterápicos, como a possibilidade de registrá-los como fitoterápicos tradicionais ou classificá-los como isentos de registro. $N$ ão que este procedimento sejustifiquepela inocuidade desta classe de produtos, visto que algumas espécies de plantas medicinais têm alta toxicidade, como exemplificado na introdução deste trabal ho.
Ao se detalhar os principais pontos observados durante a inspeção de boas práticas de fabricação nas empresas estudadas, observou-se no item administração e informações gerais, que a maioria das empresas se apresenta em situação satisfatória. Entretanto, foi constatado nas empresas avaliadas que a maioria das não-conformidades observadas se relacionava à ausência do farmacêutico responsável, denotando a falta de profissionais qualificados na empresa. Este dado seassocia novamenteà carência de investimentos por parte da direção das empresas, eainda a localização afastada dos grandes centros do estado, tornando ainda mais difícil, principalmente sob o aspecto financeiro, fixar profissionais qualificados no quadro funcional destas empresas. Uma

Tabela 1. Análise da situação sanitária e sua relação com o porte financeiro das empresas fabricantes de medicamentos fitoterápicos e oficinais no Estado do Rio de Janeiro com base na adequação de boas práticas de fabricação.

\begin{tabular}{|c|c|c|c|c|c|c|}
\hline \multirow[t]{2}{*}{ Situação sanitária } & \multirow{2}{*}{$\begin{array}{l}\text { Definição das condições } \\
\text { para cumprimento da } \\
\text { resolução RDC n } 210 / 03\end{array}$} & \multirow[t]{2}{*}{$(\%)^{\mathrm{a}}$} & \multicolumn{4}{|c|}{ Porte financeiro } \\
\hline & & & Grande & M édio & Pequeno & Micro \\
\hline Satisfatória & $\begin{array}{l}\text { Apresenta todos os itens } \\
\text { imprescindíveis } \\
+85 \% \text { dos itens necessários }\end{array}$ & 29 & 4 & 4 & 6 & - \\
\hline $\begin{array}{l}\text { Satisfatória com } \\
\text { restrições }\end{array}$ & $\begin{array}{l}\text { Apresenta todos os itens } \\
\text { imprescindíveis } \\
+50 \% \text { dos itens necessários }\end{array}$ & 10 & - & 4 & 1 & - \\
\hline Insatisfatória & $\begin{array}{l}\text { Não-observação das exigências } \\
\text { mínimas de BPF, aplicada para } \\
\text { Licença Inicial ou desinterdição; }\end{array}$ & 6 & - & - & 2 & 1 \\
\hline $\begin{array}{l}\text { parcialmente } \\
\text { Interditada }\end{array}$ & $\begin{array}{l}\text { Não-atendimento de itens } \\
\text { imprescindíveis, aplicada para } \\
\text { linhas, classes de produtos e/ou } \\
\text { atividades específicas. }\end{array}$ & & & & & \\
\hline totalmente & $\begin{array}{l}\text { Não atendimento de itens } \\
\text { imprescindíveis. Risco sanitário } \\
\text { elevado, aplicado para linhas, } \\
\text { classes de produtos e/ou } \\
\text { atividades específicas. }\end{array}$ & 40 & - & 1 & 16 & 2 \\
\hline $\begin{array}{l}\text { Processo de } \\
\text { cancelamento }\end{array}$ & - & 5 & - & - & 5 & 2 \\
\hline
\end{tabular}

a Situação sanitária das empresas analisadas ( $n=48)$. 
Tabela 2. Comparação das exi gências técnicas de registro de medicamentos fitoterápicos e alopáticos regulamentadas pela ANVISA.

\begin{tabular}{lcc}
\hline \multicolumn{1}{c}{ Exigência técnica } & Fitoterápico & Alopático \\
\hline Biodisponibilidade & Sim & Sim \\
Estabilidade & Sim & Sim \\
Equivalência & Sim & Sim \\
$\begin{array}{l}\text { Dosagem de teor } \\
\text { Dissolução em formulações } \\
\text { sólidasorais }\end{array}$ & Sim & Sim ão \\
$\begin{array}{l}\text { Registro baseado em dados } \\
\text { bibliográficos, dispensando }\end{array}$ & Sim & Não \\
$\begin{array}{l}\text { estudos de biodisponibilidade } \\
\text { Qualificação de fornecedores }\end{array}$ & Sim & Sim \\
$\begin{array}{l}\text { Testes clínicos, farmacológicos } \\
\text { e toxicológicos }\end{array}$ & Sim & Sim \\
\hline
\end{tabular}

clara predominância de inadequação sanitária frente ao porte financeiro da empresa principalmente no que concerne às empresas de pequeno porte também é observado (Tabela 1). Reforçase, portanto, a dificuldade ou até mesmo a impossibilidade de adequação destas empresas por questões claramente financeiras ou gerenciais.

Em termos gerais, a avaliação das condições das instalações mostrou que poucas apresentavam restrições, sendo a maior parte delas relacionadas ao descumprimento das diretrizes deboas práticas de fabricação na adequação ou preparação do projeto arquitetônico da planta produtiva. M uitos dos projetos analisados são adaptações de prédios destinados a outras atividades industriais para a atividade de fabricação de medicamentos. A maioria das não-conformidades detectadas estava associada à deficiência no controle de entrada de vetores, ao acesso controlado à planta produtiva, ao dimensionamento de almoxarifados e ao número e qualidade dos vestiários. Este resultado aponta para 0 amadorismo eafalta deconhecimento técnico quando da construção ou ajuste da área de fabricação das indústrias farmacêuticas consideradas como inadequadas neste segmento.

Em relação aos itens reclamações, devoluções e recolhimento, que dificilmente são considerados relevantes dentro de uma mentalidade empresarial pouco técnica e sem um suporte adequado por parte de profissionais farmacêuticos ou deáreas afins, foi possível se verificar um percentual de não-conformidades próximo a 50\% do total das empresas avaliadas. Desta forma, se torna admissível concluir que quanto mais técnico e imaterial se caracteriza a exigência de boas práticas de fabricação, menos observada esta é. Os subitens 6.4 e 6.5 da RDC n 210 são considerados imprescindíveis, visto sua relevância em termos da garantia da saúde da população e envolvimento decasos de desvio crítico da qualidade associado a um medicamento (Tabela 3). Como pode ser observado na Tabela 3, estes subitens não eram cumpridos por um grande número deempresas (27), denotando deforma ainda mais explícita a gravidade da situação. 0 sétimo tópico do Roteiro de Inspeção da Resolução RDC no 210/03 refere-se aos sistemas e instalações da unidade de tratamento de água da empresa e écomposto por sessenta itens informativos, três recomendáveis, 61 necessários e dois imprescindíveis. A análise deste tópico mostrou que a maioria das empresas (58\%) se apresentava em situação de satisfatoriedade quanto aos sistemas e instalações de tratamento de água utilizados. Porém, ainda é significativamente al to o número de empresas que se encontram insatisfatórias também neste ponto. Foi observado ainda que a maioria das empresas não completava as exigências quanto ao tópico referente ao sistema de tratamento de água. Este fato é preocupante, uma vez que a qualidade da água utilizada é um dos fatores determinantes da qualidade final do produto, principalmente devido às características dos produtos fitoterápicos e oficinais, muito susceptíveis à contaminação microbiológica mesmo na forma sólida ${ }^{16}$.

Em termos da adequação da área de produção demedicamentosfitoterápicos, foi evidenciada a falta de especial ização da área física utilizada, como no caso dos almoxarifados e a estrutura da fábrica como um todo, sendo demonstrado que $45,8 \%$ do total deempresas inspecionadasencontravam-se com mais de $15 \%$ dos itens da norma não-conformes, estando insatisfatórias, portanto, para atividade de produção de medicamentos. Em relação aos itens descritos por linhas de produção (sólidos, semi-sólidoselíquidos), foi constatado que, em geral, as não-conformidades são referentes à inadequação da documentação da qualidade, como o descumprimento com exatidão das ordens de produção, a falta de calibração e verificação de balanças e demais equipamentos de produção, a fal ta de testes de controleem processos, a ausência dos programas de qualificação e validação, a inexistência de área exclusiva para 
Tabela 3. Seção do anexo III da RDC n 210 que trata da questão referente ao recolhimento de produto onde se incidiu um maior número de não-conformidades.

\begin{tabular}{|c|c|c|}
\hline Item & Qualif & Exigência referente ao item \\
\hline 6.2 & $\mathrm{~N}$ & $\begin{array}{l}\text { A empresa possui um sistema operacional, devidamente estruturado, } \\
\text { para o recolhimento de produtos com desvio de qualidade do mercado? }\end{array}$ \\
\hline 6.3 & $\mathrm{~N}$ & Existem POPs para o recolhimento de produtos? \\
\hline 6.3 .1 & $\mathrm{~N}$ & $\begin{array}{l}\text { A empresa estabelece e mantém sistemática que garanta a correta } \\
\text { aplicação desses procedimentos? }\end{array}$ \\
\hline 6.4 & I & $\begin{array}{l}\text { Os registros correspondentes aos distribuidores permitem a } \\
\text { rastreabilidade dos produtos visando seu efetivo recolhimento? }\end{array}$ \\
\hline 6.5 & I & $\begin{array}{l}\text { No caso de recolhimento, por desvio de qualidade, as autoridades sanitárias } \\
\text { competentes são imediatamente informadas? }\end{array}$ \\
\hline 6.9 & $\mathrm{~N}$ & $\begin{array}{l}\text { São mantidos registros do recolhimento de produtos do mercado, } \\
\text { incluindo a investigação de suas causas? }\end{array}$ \\
\hline 6.10 & $\mathrm{~N}$ & $\begin{array}{l}\text { As informações disponíveis permitem determinar o percentual de recolhimento } \\
\text { do produto expedido? }\end{array}$ \\
\hline 6.11 & $\mathrm{~N}$ & Existem relatórios sobre o destino dos produtos recolhidos do mercado? \\
\hline
\end{tabular}

embalagem demedicamentos ea falta de reconciliação entre a quantidade teórica de materiais e a quantidade real utilizada na produção.

Ao se analisar o exercício de atividades diferentes da produção demedicamentos, nota-seque muitas das empresas avaliadas realizavam algumas destas atividades, tornando ainda mais complexa a adequação das mesmas à legislação sanitária atual. Muitas destas atividades devem ser realizadas obrigatoriamente em áreas segregadas; entretanto, acabavam por serem conduzidas em um mesmo local dentro das instalações estudadas. Constatou-se a utilização da mesma área, inclusive de equipamentos, para produção concomitante de ou tros produtos de natureza distinta (alimentos e/ou cosméticos), fato que contraria o preconizado na Lei Federal no 6.360/76.

A deficiência técnico-científica de boa parte do segmento é evidenciada de forma inequívoca ao se anali sar seu controle e sua garantia da qualidade. Onze empresas encontram-se com as atividades de controle de qualidade insatisfatórias e quatro destas empresas nem sequer realizavam as mesmas para os medicamentos que vinham fabricando. Na questão relacionada à garantia da qualidade, foi constatado que 22,9\% das empresas estão insatisfatórias em relação a este setor e 39,6\% ignoravam totalmente a necessidade de implantar um sistema de garantia de qualidadeem seu negócio.

Durante o período de janeiro de 2002 a abril de2004, foram recebidas eapuradas dezessete de- núncias relacionadas a classes específicas de medicamentos fitoterápicos. Destas, nove referiamse a medicamentos sem registro junto ao Ministério da Saúde, seis indicando a comercialização deprodutos por empresasnão regularizadasjunto aos órgãos sanitários competentes, uma informando sobre irregularidades cometidas por empresa com uma linha interditada, e uma informando sobre a comercialização de medicamentos de uma empresa interditada. Logo se conclui ser o sistema de farmacovigilância bastante ineficiente em relação ao medicamento fitoterápico. Esta conclusão é resultante da circulação restrita destes medicamentos na rede de hospitais sentinelas da ANVISA ea ausência de mecanismos alternativos eficientes para cobrir esta falta como a cobrança destas informações das próprias empresas fabricantes. Logo a baixa adequação destas empresas e a ausência de um efetivo monitoramento pós-registro, diferente das inspeções sanitárias, faz com que a ANVISA assuma papel ímpar na garantia da qualidade do medicamento fitoterápico comercializado no Brasil.

\section{Considerações finais}

A adequação técnica com relação aos fitoterápicos é, sem dúvida, tema de preocupação constante, pois a exposição a estes não é livre de riscos e nem sempre é eficaz para a indicação para a qual está sendo utilizado. Algumas vezes, substâncias 
sintéticas associadas e outras não declaradas fazem parte da formulação do fitoterápico, enquanto que, por outro lado, questões como a complexidade que envolve a análise deste tipo de produto, a falta de especificações para o controle de qualidade em nossas farmacopéias e a pouca fiscalização sanitária facilitam sobremaneira adulterações, contribuindo para o agravamento da baixa qualidade dos fitoterápicos e classificandoos como um problema grave de Saúde Pública. 0 problema da baixa qualidade dos fitoterápicos assume proporções imprevisíveis principalmente quando o lote defeituoso retrata a fraudeintencional, a exposição da população a riscos desconhecidos, a baixa qualidade da matéria-prima, a sonegação de informação e a inobservância da legislação sanitária. Existe uma grande contradição no fato do Brasil ser dono da maior biodiversidade do planeta e não conseguir desenvolver uma indústria fitoterápica própria, como aconteceem outros países como a China ea Índia, que inclusive exportam para o Brasil. Ao mesmo tempo, há diversos laboratórios estrangeiros paten- teando complexos fitoterápicos da flora brasileira sem que haja qualquer reação legal por parte dos órgãos competentes do governo brasileiro.

O Brasil pode vir a ser um novo e grande pólo de plantas medicinais do mundo, colocando no mercado farmacêutico substâncias processadas e com valor tecnológico agregado e não servindo como mero fornecedor de matéria-prima. Para queisto aconteça, éindispensável a adequação das empresas à legislação vigente. D esta maneira, éinequívoca a posição de quea RDC no 210 deva continuar a ser utilizada para inspecionar este segmento, não cabendo nenhum tipo de tratamento diferenciado para o setor e sim uma maior profissionalização do segmento, tanto em termos técnicos como em termos empresarias. Torna-se óbvia a situação de que as empresas que permanecerem no segmento terão de alcançar competitividade elucratividade compatíveis, ou até mesmo maiores, do que as observadas para as empresas farmacêuticas de produtos sinteticamente obtidos, devendo inclusive cumprir de forma ampla a legislação vigente no país. 


\section{Colaboradores}

ND da Costa Alves trabalhou na concepção teórica, coleta de dados e elaboração e redação final do texto; TC dos Santos trabalhou na concepção teórica e elaboração e redação final do texto; CR Rodrigues trabalhou na concepção teórica e elaboração e redação final do texto; HC Castro trabalhou na concepção teórica e elaboração e redação final do texto; LM Lira trabalhou na concepção teórica, coleta de dados e elaboração e redação final do texto; CB Dornelas trabalhou na concepção teórica, coleta de dados e elaboração e redação final do texto e LM Cabral trabaIhou na concepção teórica e elaboração e redação final do texto.

\section{Referências}

1. Akerele 0 . Nature's medicinal bounty: don't throw it away. World Health Forum1993; 14:390-395.

2. Bijos GM, Pillar O. Legislação farmacêutica (de 1931 a 1964). Rio de Janeiro: São José; 1964.

3. Bove GM. Acute neuropathy after exposure to sun in a patient treated with St John's wort. Lancet 1998; 352:1121-1122.

4. Brasil. Ministério da Saúde. Agência Nacional de Vigilância Sanitária. Resolução RDC no 48 de 16 de março de 2004. Dispõe sobre o registro de medicamentos fitoterápicos. Diário Oficial da União 2004; $18 \mathrm{mar}$.

5. Capasso R, Izzo AA, Pinto L, Bifulco T, Vitobello $C$, Mascolo N. Phytotherapy and quality of herbal medicines. Fitoterapia 2000; 71:S58-S65.

6. Cole G. Pharmaceutical production and facilities: design and applications. New York: Ellis Horwood Editors; 1998.

7. Ernst E, Pittler MH. Risks associated with herbal medicinal products. Wien Med Wochenschr 1998; 152:183-189.

8. Guil JL, Rodriguez-Garcia I, Torija E. Nutritional and toxic factors in selected wild edible plants. Plant Foods Hum Nutr 1997; 51:99-107.

9. Maluf $E$, Barros $H M T$, Frochtengarten $M L$, Benti $R$, Leite Jr. Assessment of the hypnotic sedative effects and toxicity of Passiflora-edulis aqueous extract in rodents and humans. Phytotherapy Res 1991; 5:262266.
10. Miller LG. Herbal medicinals: selected clinical considerations focusing on known or potential drugherb interactions. Arch Intern M ed 1998; 158:22002211.

11. Schaller M, Korting HC. Allergic airborne contact dermatitis from essential oils used in aromatherapy. Clin Exp Dermatol 1995; 20:143-145.

12. Schelosky L, Raffaut C, Jendroska K, Poewe W. Kava and dopamine antagonism. J Neurol Neurosurg Psychiatry 1995; 58:639-640.

13. Schneck C. St. John's wort and hypomania. J Clin Psychiatry 1998; 59:689-692.

14. Simões M O, Schenkel EP, Gosmann G, M ello JCP, M entz LA, Petrovick PR. Farmacognosia: da planta ao medicamento. Porto Alegre/Florianópolis: Editora da UFRGS/Editora da UFSC; 1999.

15. Veiga VR, Pinto AC, M aciel MAM. M edicinal plants: safe cure? Quím. N ova 2005; 28:519-528.

16. Wijesekera RO. The medicinal plant industry. Flórida: CRC Press; 1991

17. Wong AHC, Smith M, Boon HS. Herbal remedies in psychiatric practice. Arch Gen Psychiatry 1998; 55:1033-1044.

18. Yunes RA, Pedrosa, RC, Cechinel-Filho V. Fármacos e fitoterápicos: a necessidade do desenvolvimento da indústria de fitoterápicos e fitofármacos no Brasil. Quím. Nova 2001; 24:147-152.

Artigo apresentado em 08/02/2007

Aprovado em 14/01/2007 